bioRxiv preprint doi: https://doi.org/10.1101/2022.02.15.480494; this version posted February 17, 2022. The copyright holder for this preprint (which was not certified by peer review) is the author/funder, who has granted bioRxiv a license to display the preprint in perpetuity. It is made available under aCC-BY-NC-ND 4.0 International license.

\title{
1 Strain-dependent induction of primary bile acid 7-dehydroxylation by cholic acid
}

\section{Authors}

3 Eduard Vico-Oton ${ }^{1}$, Colin Volet ${ }^{1}$, Nicolas Jacquemin ${ }^{1}$, Karin Lederballe Meibom ${ }^{1}$, Rizlan Bernier4 Latmani $^{{ }^{*}}$

5

6

7

8

${ }^{1}$ Environmental Microbiology Laboratory, School of Architecture, Civil and Environmental Engineering, École Polytechnique Fédérale de Lausanne, Lausanne, Switzerland.

*Corresponding author. E-mail address rizlan.bernier-latmani@epfl.ch. Postal address EPFL ENAC IIE EML CH A1 375 (Bâtiment CH) Station 6 CH-1015 Lausanne Switzerland. Tel. +41 216935001.

\section{Abstract (250 words)}

Bile acids (BAs) are steroid-derived molecules with important roles in digestion, the maintenance of host metabolism and immunomodulation. Primary BAs are synthesised by the host and their concentrations tightly regulated, while secondary BAs are produced by the gut microbiome through transformation of the former. Regulation of microbial production of secondary BAs is not well understood, particularly the production of 7-dehydroxylated BAs, which are the most potent agonists for host BA receptors. The 7-dehydroxylation of cholic acid (CA) is well established and is linked to the expression of a bile acid-inducible (bai) operon. However, little to no 7-dehydroxylation has been reported for other host-derived BAs (e.g., chenodeoxycholic acid, CDCA or the mouse ursodeoxycholic acid, UDCA) and it is unknown whether they induce bai gene expression. Here, we demonstrate that the 7-dehydroxylation of CDCA and UDCA by Clostridium scindens is induced by CA suggesting that CAdependent transcriptional regulation of 7-dehydroxylation is generalisable to CDCA and UDCA. Nevertheless, this effect is strain-specific and the murine isolate Extibacter muris did not respond to CA exposure, suggesting that bai genes are regulated differently in this strain. Moreover, the accessory gene bail was only upregulated in the Clostridium scindens ATCC 35704 strain, implying mechanistic differences amongst isolates. Interestingly, the $C$. scindens strains were also capable of 7-DH-ing muricholic acids (MCAs) to a limited extent. This study shows novel 7-dehydroxylation activity in vitro as a result of CA-driven induction and suggests that it is likely that a different BA plays the same role in the murine gut.

\section{Keywords}

7-dehydroxylation, Clostridium scindens, Extibacter muris, gut microbe, deoxycholic acid (DCA), lithocholic acid (LCA), muricholic acid (MCA), CA co-induction, bai gene expression, conjugated bile acids

\section{Introduction}

Primary bile acids (BAs) are metabolites synthesised from cholesterol by hepatocytes while secondary BAs are produced by the gut microbiome through the transformation of primary BAs (Figure 1). In the liver, the BA are conjugated to glycine or taurine. The three main microbial BA transformations are deconjugation (loss of the amino acid group), oxidation (of one or several of the hydroxyl groups), and $7 \alpha$-dehydroxylation (7-DH-ion), the loss of a hydroxyl group at the $\mathrm{C} 7$ position ${ }^{1}$. These microbial transformations increase the diversity of the BA pool (Figure 1) and determine BA affinity to host receptors. In particular, 7-DH-ion turns primary BAs such as cholic acid (CA) and chenodeoxycholic acid (CDCA) into the 7-dehydroxylated (7-DH-ed) BAs deoxycholic acid (DCA) and lithocholic acid (LCA), respectively ${ }^{2}$. 
BAs act as detergents to solubilise dietary fats, but also have important metabolic and immunomodulatory roles through activation of their target receptors ${ }^{3}$. The two best-studied BA receptors are the Farnesoid X Receptor (FXR), a nuclear receptor, and the G Protein-Coupled Bile Acid Receptor, GPBAR1, also known as Takeda G-Protein Receptor 5 (TGR5), which is a membrane receptor. FXR is activated through the binding of BA agonists, particularly the 7-DH-ed LCA but also DCA ${ }^{4}$. FXR activation results in the inhibition of primary $B A$ synthesis through repression of the cholesterol $7 \alpha-$ hydroxylase CYP7A1. Regulation of BA production limits BA concentration and therefore, toxicity. On the other hand, BA dysregulation can cause health issues such as cholestasis, Irritable Bowel Syndrome (IBS), gallstone disease, or even the induction of colon cancer ${ }^{5-7}$. Besides BA homeostasis, FXR also has focal roles in glucose and lipid homeostasis ${ }^{8}$. Similarly, TGR5 is a multifunctional regulator involved in glucose homeostasis, energy expenditure, and the modulation of the inflammatory response ${ }^{9,10}$. LCA, DCA and their tauro-conjugated forms TLCA and TDCA, are among the strongest agonists of TGR5 ${ }^{11,12}$, highlighting the importance of microbial transformation, particularly 7-DH-ion, in TGR5 activation. Moreover, DCA and LCA have protective properties against Clostridium difficile infection ${ }^{13,14}$.

The study of BAs has traditionally been based on mouse models ${ }^{15}$. Besides CA and CDCA, mice (and other rodents) also generate muricholic acids (MCAs) such as $\alpha$-MCA and $\beta$-MCA (Figure 1 ) and rehydroxylate DCA and LCA in the liver ${ }^{16}$. Additionally, the mouse liver is capable of producing primary ursodeoxycholic acid (UDCA) ${ }^{16}$ although there is evidence that the gut microbiome is responsible for a significant fraction of UDCA in the gut ${ }^{14}$ (Figure 3 in manuscript provided for reviewers). On the other hand, UDCA is exclusively a secondary BA in humans ${ }^{7}$. Because 7-DH-ion plays a major role in host homeostasis, significant effort has been expended to study 7-dehydroxylating (7-DH-ing) bacteria.

Nonetheless, experimental evidence of 7-DH-ion is limited to a few species of the Clostridiales order. One of the best characterised is the human isolate Clostridium scindens ATCC 35704, the type strain of C. scindens ${ }^{17}$. The Extibacter muris DSM 28560 (JM40) strain was recently isolated from mice and identified as a 7-DH-ing organism ${ }^{18,19}$. The ability of $C$. scindens ATCC 35704 to 7-dehydroxylate both in vivo and in vitro is well established ${ }^{20}$; E. muris DSM 28560 has also been shown to 7-dehydroxylate in vivo, transforming primary $B A s C A, C D C A, \alpha M C A, \beta M C A$ and UDCA into their respective secondary BAs DCA, LCA and MDCA (Figures 3 and 5 in manuscript provided for reviewers).

The biochemical machinery for 7-DH-ion is encoded in the bai (bile acid inducible) eight-gene operon (baiBCDEA2FGHI) $)^{21}$. In addition, the $C$. scindens ATCC 35704 strain harbours the accessory gene baiJ (HDCHBGLK_03451) ${ }^{22}$ whereas the E. muris DSM 28650 genome includes a baiJKL pseudogene cluster $^{23}$. Most of the published work on the 7-DH-ion pathway has been performed with another $C$. scindens strain, VPI $12708^{24,25}$. A recent publication by Funabashi et al. cloned the bai operon of $C$. scindens VPI 12708 into Clostridium sporogenes which then showed in vivo 7-DH-ing activity 25. Different strains exhibit varying efficiency in 7-DH-ing CA in vitro. The C. scindens ATCC 35704 and VPI 12708 strains show almost complete transformation to DCA while E. muris DSM 28560 has more limited activity ${ }^{19,20,26}$. Other known 7-DH-ing strains such as Clostridium hylemonae and Peptacetobacter hiranonis have been reported as harbouring weak and strong activity, respectively ${ }^{27}$ and a new strain of $P$. hiranonis recently isolated from dog faeces displayed in vitro 7-DH-ion at around $30 \%$ conversion of $C A$ to $D C A^{28}$. Notably, in vitro 7-DH-ion of other primary BAs has been reported to be minor (CDCA) or non-existent (MCAs and UDCA) ${ }^{20,23,29}$.

The limited in vitro 7-DH-ion of primary BAs other than CA (i.e., CDCA and MCAs) is striking considering that secondary 7-DH-ed forms of these BAs are routinely detected at significant concentrations in the host $^{30,31}$. Most studies tackling in vitro primary bile acid 7-DH-ion consider each BA in isolation. In addition, significant overexpression of the bai operon in response to CA has been reported ${ }^{32,33}$ but 
bioRxiv preprint doi: https://doi.org/10.1101/2022.02.15.480494; this version posted February 17, 2022. The copyright holder for this preprint (which was not certified by peer review) is the author/funder, who has granted bioRxiv a license to display the preprint in perpetuity. It is made available under aCC-BY-NC-ND 4.0 International license.

there is no information about the potential induction of this operon by other primary BAs (i.e., CDCA and MCAs) and whether induction by CA also results in the transformation of the latter. We hypothesise that $\mathrm{CA}$-dependent induction of the bai operon promotes 7-DH-ing activity of other BAs when they occur together with CA. Moreover, we posit that primary BAs other than CA cannot induce their own transformation.

Here, the expression of bai genes was measured in vitro in the presence of CA, CDCA, $\alpha M C A, B M C A$ and UDCA, with and without co-induction with CA to test whether the overexpression of bai genes was exclusive to CA and whether CA-induced overexpression was sufficient to promote the 7-DH-ion of other BAs. The experiments were performed with three strains, the human isolates $C$. scindens ATCC 35704 and VPI 12708 and the murine isolate E. muris DSM 28561 (SJ24). The results show that the response to CA co-induction was strain-variable. It was highly effective for $C$. scindens strains and sufficient to promote the transformation of other primary BAs.

This work highlights the importance of the presence CA for the 7-DH-ion of other BAs. Moreover, the lack of activity from $E$. muris SJ24 points at potential host-related differences whereby CA may not be the key inducer for BA 7-DH-ion in the murine gut.

\section{Results}

\section{In vitro bile acid transformation and impact of ${ }^{13} \mathrm{C}-\mathrm{CA}$ co-induction}

Two human isolates C. scindens ATCC 35704 and C. scindens VPI 12708 and one murine isolate E. muris DSM 28561 (SJ24) were tested for their ability to 7-dehydroxylate human and mouse primary BAs in vitro. Moreover, parallel experiments were performed by amending the cultures with ${ }^{13} \mathrm{C}-\mathrm{CA}$ in order to test whether the 7-DH-ion of CDCA, $\alpha M C A, B M C A$ and UDCA could be induced by CA (Supplementary Table 1). As expected, all three strains 7-DH-ed CA but to varying extents (Figure 2). C. scindens ATCC 35704 and C. scindens VPI 12708 showed strong 7-DH-ing activity with $97 \%$ and $80 \%$ CA conversion to 7-DH-ed BAs after 48 hours, respectively. E. muris SJ24, on the other hand, only converted $9 \%$ of the CA provided into 7-DH-ed forms (Figure 2). Similar results were obtained when this experiment was repeated as part of the uninduced batch of conditions (Supplementary Figure 1). C. scindens ATCC 35704 produced up to $52.26 \mu \mathrm{M}$ DCA after 32 hours. Some of the DCA was subsequently oxidised to 12-oxolithocholic acid (12-oxoLCA) (Figure 2A). In contrast, $C$. scindens VPI 12708 produced the highest amount of DCA after 48 hours $(71.42 \mu \mathrm{M})$, with little to no oxidised DCA forms $(0.19 \mu \mathrm{M}$ of 3-oxoDCA at 48 hours) (Figure 2B). The lack of 12 -oxo forms from the $C$. scindens VPI 12708 strain was expected since the $12 \alpha$-hydroxysteroid dehydrogenase (12 $\alpha$-HSDH) required for this process was not detected by PCR in this strain (the full genome is currently unavailable) (data not shown). Finally, E. muris SJ24 only produced $8.1 \mu \mathrm{M}$ of DCA after 48 hours with very low amounts of oxidised forms of DCA ( $0.21 \mu \mathrm{M}$ of 12-oxoLCA) (Figure 2C). It is important to highlight that E. muris does not possess a $3 \alpha-H S D H$ encoded by baiA2 which was recently identified as an important component of the CA 7-DH-ion pathway ${ }^{25}$. This strain may perform hydroxyl group oxidations at the C3 position using BaiA1/3 which has lower affinity to CA than BaiA2 ${ }^{34}$ and is present outside the bai operon ${ }^{35}$. All three strains also showed a modicum of 7-oxidation activity (Figure 2), resulting in the formation of 7-oxoDCA, which cannot be 7-DH-ed.

CDCA $7 \alpha$-dehydroxylation is limited for all three strains. Indeed, $C$. scindens ATCC 35704 only produced $1.59 \mu \mathrm{M}$ LCA and C. scindens VPI 12708 only $1.55 \mu \mathrm{M}$ LCA (Figure 3AB), whereas for E. muris, no LCA was detected. The latter is in line with previous reports ${ }^{23}$. The amendment of ${ }^{13} \mathrm{C}$-CA significantly increased the transformation of CDCA for both $C$. scindens strains ( $p$-value $<0.001$ two-way ANOVA) but had no impact on E. muris SJ24 (Figure 3C). Indeed, the LCA yield increased to $9.77 \mu \mathrm{M}$ for strain 
bioRxiv preprint doi: https://doi.org/10.1101/2022.02.15.480494; this version posted February 17, 2022. The copyright holder for this preprint (which was not certified by peer review) is the author/funder, who has granted bioRxiv a license to display the preprint in perpetuity. It is made available under aCC-BY-NC-ND 4.0 International license.

ATCC 35704 and to $40.4 \mu \mathrm{M}$ for strain VPI 12708 (Figure 3AB). No change was observed for E. muris SJ24.

As for UDCA, $7 \alpha$-dehydroxylation to LCA was observed only upon amendment with ${ }^{13} \mathrm{C}$-CA (Figure 4). None of the strains exhibited any detectable level of activity from cultures that included only UDCA. $14.92 \mu \mathrm{M}$ of LCA as well as extremely low amounts of 3-oxoLCA (with a maximum of $0.12 \mu \mathrm{M}$ at 32 hours) were detected when $C$. scindens ATCC 35704 was co-induced. An unknown oxidised form labelled X-oxoUDCA was detected with a maximum concentration of $2.41 \mu \mathrm{M}$ after 32 hours (Figure $4 A$ ). It is likely that this BA corresponds to 3-oxoUDCA (3-oxo-7 $\beta$-Hydroxy-5 $\beta$-cholan-24-oic acid) as we can exclude 7-oxoLCA (the other product of oxidation of UDCA) (Figure 1). Another BA with the same ionised mass as UDCA was detected at a maximum concentration of $4.24 \mu \mathrm{M}$ after 24 hours. We propose that this could be an isoform of UDCA with the hydroxyl group of the C3 carbon in $\beta$ conformation ( $3 \beta, 7 \beta$-Dihydroxy-5 $\beta$-cholan-24-oic acid). However, the identity of these compounds remains unconfirmed due to the lack of standards. Upon co-induction, the 7-DH-ing activity of $C$. scindens VPI 12708 was comparable to that of the co-induced ATCC strain, with $13.61 \mu \mathrm{M}$ of LCA and $0.68 \mu \mathrm{M}$ of 3-oxoLCA after 48 hours. X-oxoUDCA was also detected at very small concentrations around $0.5 \mu \mathrm{M}$ from 12 hours until the end of the experiment. The potential isoform of UDCA was detected at up to $9.92 \mu \mathrm{M}$ at the 24-hour time point. Following the same trend observed with the other primary BAs, E. muris SJ24 did not show any detectable activity with UDCA even after coinduction. The chromatograms for the unknown bile acids can be found in Supplementary Figure 2 .

As expected, neither $C$. scindens strain nor $E$. muris were capable of $\alpha \mathrm{MCA} 7 \alpha$-dehydroxylation in the absence of ${ }^{13} \mathrm{C}$-CA (Figure 5). C. scindens ATCC 35704 only produced minute amounts of an unknown oxo form of aMCA (labelled Y-oxo $\alpha \mathrm{MCA})\left(0.85 \mu \mathrm{M}\right.$ at 32 hours). Once co-induced with ${ }^{13} \mathrm{C}-\mathrm{CA}, 6-$ oxoMDCA was detected at $2.7 \mu \mathrm{M}$ after 48 hours in the $C$. scindens ATCC 35704 culture (Figure 5A). This secondary bile acid has been $7 \alpha-\mathrm{DH}$-ed but also the hydroxyl at $\mathrm{C} 6$ oxidised. Moreover, several intermediates for which standards are unavailable were also detected after 48 hours. These were unknown oxidised forms of $\alpha$ MCA (labelled X-and Y- oxo $\alpha M C A$ ) at concentrations not exceeding $5 \mu \mathrm{M}$ each. A third unknown BA was detected (albeit at very low concentrations, $0.41 \mu \mathrm{M}$ at 32 hours) with the same mass as 6-oxoMDCA, suggesting that it is an MCA species with one oxidation and one dehydroxylation. This would indicate the production of another $7 \alpha$-dehydroxylated form of $\alpha \mathrm{MCA}$ in vitro (Figure 1, Figure 5A). As for the ATCC 35704 strain, C. scindens VPI 12708 exhibited an increase in the quantity of products from $\alpha \mathrm{MCA}$ transformation in the presence of ${ }^{13} \mathrm{C}$-CA relative to its absence (Figure 5B). This includes the 7-DH-ed BA 6-oxoMDCA that reached a concentration of $8.18 \mu \mathrm{M}$ after 48 hours and the $X$ - and $Y$ - $\alpha M C A$ forms that were detected at maximum concentrations of $4.22 \mu \mathrm{M}$ (4 hours) and $1.69 \mu \mathrm{M}$ (32 hours), respectively. The aforementioned aMCA-derived bile acid with one ketone group and one dehydroxylation was also detected at a maximum concentration of $3.49 \mu \mathrm{M}$ after 48 hours (Figure 5B). Surprisingly, E. muris SJ24 did not exhibit any observable 7-DH-ing activity with or without ${ }^{13} \mathrm{C}$-CA. Nevertheless, a small amount of X-oxoaMCA was detected at all time points, with a stable concentration at around $2.4 \mu \mathrm{M}$ without and $1.9 \mu \mathrm{M}$ with ${ }^{13} \mathrm{C}-\mathrm{CA}$ (Figure $5 \mathrm{C}$ ). The results for $B M C A$ were very similar to those for $\alpha M C A$ and are discussed in further detail in the supplementary information.

The concentration of ${ }^{13} \mathrm{C}$-CA was also measured over time to ascertain that $\mathrm{CA}$ was being metabolised. It was observed to decrease until disappearing after 48 hours in the $C$. scindens strains except in the presence of CDCA, for which the concentration decreased slowly over time. We attribute this observation to the toxicity of CDCA in that concentration ${ }^{20}$. On the other hand, the concentration of ${ }^{13} \mathrm{C}-\mathrm{CA}$ in E. muris remained stable over time and in all conditions (Supplementary Figure 3). 
bioRxiv preprint doi: https://doi.org/10.1101/2022.02.15.480494; this version posted February 17, 2022. The copyright holder for this preprint (which was not certified by peer review) is the author/funder, who has granted bioRxiv a license to display the preprint in perpetuity. It is made available under aCC-BY-NC-ND 4.0 International license.

Induction of bai genes expression in the presence of primary bile acids

In order to assess whether the amendment of ${ }^{13} \mathrm{C}-\mathrm{CA}$ to the culture induced the bai operon as hypothesised, the relative expression of baiCD and of baiE were measured. In addition, the expression of bais (an accessory gene) was also monitored. Gene expression was normalised using at least three reference genes and was calculated relative to the expression levels in a control group without BAs. Both E. muris strains, that is JM40 (DSM 28560) and SJ 24 (DSM 28561), have a truncated baiJ gene ${ }^{23}$. However, while the baiJ pseudogene is interrupted by stop codons in strain JM40, it is not in strain SJ24, making it worthwhile to investigate baiJ in the latter strain (as was done in this study). Additionally, baiO was also analysed for E. muris SJ24 as an alternative accessory bai gene ${ }^{36}$.

Results show that the expression of bai operon genes in both $C$. scindens strains was highly upregulated as a response to exposure to CA or to CDCA but not to the other BAs (Figure 6). CA and CDCA are also the only two primary BAs for which in vitro 7-DH-ion data are already available ${ }^{20}$. Moreover, it is worth highlighting that CDCA was tested using a concentration of $200 \mu \mathrm{M}$ vs. $100 \mu \mathrm{M}$ for CA.

For $C$. scindens ATCC 35704, the three genes tested were highly upregulated when ${ }^{13} \mathrm{C}$-CA was present along with another BA (UDCA, $\alpha M C A$, or $\beta M C A$ ) (Figure $6 A$ ). In the CDCA dataset, statistically significant differences relative to the uninduced conditions were observed and all genes were slightly more upregulated in the presence of ${ }^{13} \mathrm{C}-\mathrm{CA}$ ( $p$-value $<0.001$ linear model), but baiCD was more so than the other genes (Figure 6A). Most interestingly, none of the other primary BAs activated the expression of bai genes on their own, consistent with the lack of 7-DH-ing activity with these BA substrates alone. However, the expression was brought up to levels higher than those observed for CA once co-induced (Figure 6A).

A similar pattern was observed for $C$. scindens VPI 12708 but with the significant difference being that baiJ was not overexpressed under any conditions (Figure 6B) ( $p$-value $<0.001$ linear model). In the CDCA dataset, the co-induction with ${ }^{13} \mathrm{C}$-CA had an upregulatory effect if assessed with a paired Wilcoxon test (Supplementary Figure 4) but this effect was not found to be significant when using the linear statistical model displayed in Figure 6 . It is worth noting that the expression data were obtained from the mid- to late-log exponential phase (around 18 hours) when differences in activity between uninduced and co-induced conditions were not very large (Figure 3B). As with C. scindens ATCC 35704, co-induction had a dramatic effect on the expression levels of baiCD and baiE in the presence of UDCA, $\alpha M C A$, or $B M C A$, with upregulation reaching the expression levels observed with CA or CDCA alone (Figure 6B).

E. muris $\mathrm{SJ} 24$ showed a slight upregulation of baiCD and baiE in the CA dataset but it was not significant and did not translate to any of the other conditions (Figure $6 \mathrm{C}$ ), mimicking its very poor 7-DH-ing activity (Figure 2). As a matter of fact, the increased baiCD and baiE gene expression ratio observed in the CA group was probably caused by one of the biological replicates which had a higher expression level than the others.

Thus, CA had a large effect on bai expression which was strain specific. Genes of the bai operon in the two C. scindens strains (ATCC 35704 and VPI 12708) exhibited a similar response to CA induction but the accessory bail differed in its response. It was upregulated in strain ATCC 35704 but not in strain VPI 12708. In contrast, CA had no significant effect on the expression of all the bai genes considered in E. muris SJ24.

The rhaS_1 gene (HDCHBGLK_01429) is immediately upstream of the bai operon promoter on the opposite strand and has also been proposed as bile acid-regulatory (bar) due to its potential 
implication in bai regulation 7 . The expression of rhaS1 and rhaS2 (a copy of rhaS1 elsewhere in the genome) was shown to have background levels across BAs (Supplementary Figure 5). This was tested in C. scindens ATCC 35704 without the amendment of ${ }^{13} \mathrm{C}$-CA. Results indicate that rhaS is not upregulated by any of the tested BAs.

\section{Discussion}

Bile acid chemistry is a relevant field in human and veterinary medicine not only because of BA detergent function during digestion but also for the wide range of roles related to host physiology and homeostasis ${ }^{37,38}$.

The bai operon was originally described in C. scindens VPI 12708 in 1990 and this strain has become the reference for biochemical studies of the 7-DH-ion pathway ${ }^{21,35,39}$. However, genetic differences occur in the bai operon amongst 7-DH-ing strains ${ }^{35,40}$. Moreover, the presence of accessory bai genes with as-of-yet undefined roles varies across strains ${ }^{35,39}$.

Out of all primary BAs, only CA has been consistently reported to be 7-DH-ed in vitro, with low levels of 7-DH-ion for CDCA and none for other murine primary BAs ( $\alpha M C A, \beta M C A$ or UDCA) $20,23,41-44$. We confirm that the two $C$. scindens human isolates upregulated the expression of bai operon genes in response to CA (Figure 6). However, we observed differences between the two strains in the response of baiJ. This accessory gene is annotated as an urocanate reductase and a member of the oxidoreductase family. Current pathway data indicate that the gene does not play a significant role in 7-DH-ion of CA in strain VPI $12708^{25}$, which would align with the lack of expression observed from our data. In contrast, the high levels of upregulation in the ATCC 35704 strain match previously published data $^{32}$ and suggest that baiJ may play a role in the 7-DH-ion of CA or other BAs in that strain. On the other hand, E. muris SJ24, the murine isolate, barely registered any response to CA, nor activity in general, highlighting the potential differences in the 7-DH-ion pathway between human and mouse isolates.

UDCA is the $7 \beta$ isomer of CDCA and is reported to have significant therapeutic properties ${ }^{2,7,45}$. In humans, UDCA is synthesised from CDCA by gut microbes containing $7 \alpha$ - and $7 \beta-\mathrm{HSDHs}^{7,26,46,47}$ and is thus, a secondary $\mathrm{BA}$; in mice, it is produced by the liver, thus, it is a primary $\mathrm{BA}$, and is used as a precursor for $\beta M C A^{15,16}$. Nevertheless, colonisation of mice lacking any 7-dehydroxylators with 7-DHing bacteria increases UDCA levels, implying that the gut microbiome also plays a significant role in the UDCA production of mice ${ }^{14}$ (Studer et al., and appended manuscript Figures 3 and 5). Despite its importance, little is known about the capacity of bacteria to $7 \beta$-dehydroxylate UDCA in vitro and previous data have shown no activity by $C$. scindens strain ATCC $35704^{20}$. In accordance with our hypothesis, co-induction with ${ }^{13} \mathrm{C}$-CA not only greatly upregulated bai gene expression (Figure 6B) but also provided evidence of UDCA $7 \beta$-dehydroxylation as LCA was detected in the culture (Figure 4). The absence of CDCA in the culture suggests that UDCA was not 7-epimerised to CDCA prior to its $7 \alpha$ dehydroxylation. Moreover, two unidentified compounds were detected, providing further evidence of the $7 \beta$-dehydroxylation of UDCA and its associated unique set of intermediates. The oxidised intermediate is very likely to be 3-oxoUDCA (3-oxo-7 $\beta$-Hydroxy-5 $\beta$-cholan-24-oic acid), as based on the mass, the only alternative would have been 7-oxoLCA, which was quantified (Figure 4). The other unknown compound had the same mass as UDCA albeit a different retention time, suggesting this could be an iso- form of UDCA. It follows that this would be $3 \beta$-UDCA, tentatively named isoUDCA

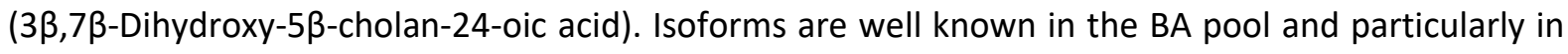
CDCA-related intermediates ${ }^{20,25,33,48}$ so it is likely that UDCA follows a similar pattern. Despite the production of unknown intermediates, the activity response to co-induction suggests that UDCA 7 $\beta$ dehydroxylation uses the same bai machinery as the 7-DH-ion of CDCA. 
The BA pool of mice is significantly more diverse than that of humans due to the primary production of muricholic acids (with a hydroxyl group at the C-6 position) and of UDCA. Their secondary bile acid pool includes DCA and LCA but also MDCA and its $6 \alpha$ counterpart, hyodeoxycholic acid (HDCA). Furthermore, mice can rehydroxylate TDCA back into TCA in the liver ${ }^{15}$. In general, the secondary BAs derived from muricholic acids seem to be in low abundance in the gut, hinting at the difficulty in 7$\mathrm{DH}$-ing these BAs. This is perhaps the reason why primary BAs such as BMCA are dominant in the bile acid pool ${ }^{49}$. While we initially hypothesised that the lack of activity was due to the lack of expression, our co-induced data show that bai gene expression in the human isolates is not sufficient for the production of MDCA. ${ }^{23}$. On the other hand, E. muris showed no significant expression.

The human isolates showed marginal activity for aMCA and BMCA upon co-induction. 7-DH-ed forms such as 6-oxoMDCA were detected (Figure $5 \&$ Supplementary Figure 6 ) but the full 7-dehydroxylation to MDCA was not observed. These results imply that other elements besides the bai operon (and baiJ) might be needed to completely 7-dehydroxylate MCAs. A potential reason could be a missing gene such as a $6 \beta-H S D H$ required for complete 7-DH-ion.

The mouse isolate $E$. muris was a weak CA 7-dehydroxylator and had no activity with the other BAs. This was coupled with a complete lack of gene upregulation in response to any BA. Nonetheless, recently published in vivo data from Studer et al., 2022 (appended manuscript Figure 3) quantified MDCA as a direct result of $E$. muris colonisation, demonstrating that this strain is capable of 7-DH-ion of MCA in vivo. To explain these differences, it is possible that an untested BA could be relevant for inducing 7-DH-ion of MCAs. An option could be TBMCA, but conjugated forms were not tested here. Both of these BAs are highly abundant in the BA pool of mice ${ }^{16}$. Finally, experimental conditions might have not been optimal to stimulate the 7-DH-ing activity ${ }^{50}$.

The novel activity for aMCA and BMCA yielded several intermediate BAs that we were not able to fully characterise due to the lack of appropriate standards. All human and mouse BAs share a backbone of four rings (Figure 1), this makes mass fractionation in the mass spectrometer unsuitable for identification. Therefore, we currently rely on comparison of ionised mass and retention time to standards. However, several assumptions can be made to speculate what these compounds could be. MCAs oxidised at the C-3, C-6 or C-7 position (Figure 1). A C-7 oxidation would yield 7-oxoMDCA regardless of the primary MCA. Meanwhile, other oxidations would be differentiated by the $\alpha$ or $\beta$ conformation of the C-7 hydroxyl. It is possible that one of the intermediates that we detected was 7oxoMDCA but none shared retention times across MCAs, meaning that different intermediates were produced for each of the two MCA substrates (Supplementary Figure 2). Considering that two oxidised intermediates are detected from both MCAs, we hypothesise that those are the 3-oxo and 6-oxo forms

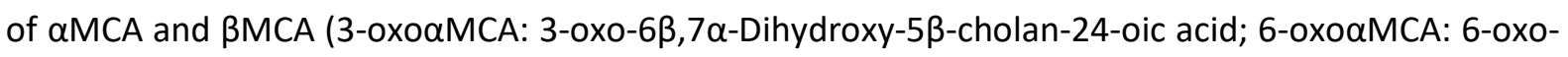
$3 \alpha, 7 \alpha$-Dihydroxy-5 $\beta$-cholan-24-oic acid; 3-oxo $\beta M C A$ : 3-oxo- $6 \beta, 7 \beta$-Dihydroxy-5 $\beta$-cholan-24-oic acid; and 6-oxo $\beta$ MCA: 6-oxo-3 $\alpha, 7 \beta$-Dihydroxy-5 $\beta$-cholan-24-oic acid). A third intermediate was also detected from both MCAs, with the ionised mass corresponding to secondary BAs with one dehydroxylation and a ketone group (e.g., 7-oxoLCA) (Figure 5 \& Supplementary Figure 6). Three options are plausible: 1 ) A dehydroxylation at the $C-3$ position. This would yield a novel family of BAs with a $6 \beta$ - and $7 \alpha / \beta$ - hydroxyls which is highly unlikely as it would have been identified previously by the multiple studies investigating the murine $B A$ pool ${ }^{15,16,51-54}$. 2) An oxidation paired with a 6dehydroxylation would yield 3-oxoCDCA, 7-oxoLCA, or 3-oxoUDCA. 3-oxoCDCA and 7-oxoLCA were included in our analysis (Supplementary Table 3) and would have been detected if present. Meanwhile, the retention time of this compound is distinct from that of the compound proposed to be 3-oxoUDCA from the transformation of UDCA (see above and Supplementary Figure 2). Thus, this is not likely to be 3-oxoUDCA. 3) A 7-dehydroxylation could allow for ketone groups at the C-3 and C- 
3126 positions. 6-oxoMDCA was available as a standard but the other option, 3-oxoMDCA (3-oxo-6 $\beta$ -

313 Hydroxy-5 $\beta$-cholan-24-oic acid), was not. It is therefore possible that this compound corresponds to

314 3-oxoMDCA, but this remains unconfirmed.

\section{Conclusion}

316 The findings presented here are threefold. First and foremost, we demonstrated that the previously

317 reported ${ }^{32,33}$ strong upregulation of bai genes by CA increases the extent of 7-DH-ion of other primary

318 BAs. This was particularly true for UDCA which has been reported to be converted to LCA in vitro for

319 the first time. Secondly, the upregulation of bai genes exhibited strain-specific differences. $C$. scindens

320 ATCC 35704 upregulated the bai operon genes baiCD and baiE as well as the bai accessory gene baiJ.

321 While for $C$. scindens VPI 12708, it upregulated the bai operon genes, but bail expression was found

322 to be at a background level. This is consistent with the lack of involvement of bail in 7-DH-ion in strain

$323 \mathrm{VPI} 12708^{25}$. E. muris SJ24 was the third strain tested, a murine isolate with in vivo 7-DH-ing capabilities

324 (appended manuscript Figure 3 and 5). Our findings with the SJ24 strain showed weak 7-DH-ion of CA

325 and no upregulation of any of the bai genes tested. The lack of activity of this murine strain even in

326 the presence of murine primary BAs suggests that there is a missing factor, maybe an untested BA or

327 other co-factors, required for an efficient bai expression and 7-DH-ing activity. It is also possible that

328 7-DH-ion of MCAs occurs through a distinct pathway with different enzymatic requirements. Finally,

329 C. scindens human isolates can partially 7-dehydroxylate MCAs. Nevertheless, there might be missing

330 a key component such as a 6-BHSDH that would complete the reductive arm of 7-DH-ion to MDCA.

331 In conclusion, these data provide novel insights into the intricacies of 7-DH-ion and the significant differences amongst 7-DH-ing bacteria. The CA-dependent co-induction can be attributed to the abundance of this compound in the BA pool of humans and mice. However, the inducing factor for $E$. muris activity remains elusive. Moreover, multiple novel BAs were observed and their identity surmised based on mass spectrometry information. Further work is required to investigate the 7-DHion pathway of CDCA, $\alpha M C A, B M C A$ and UDCA as well as exploring the strain-specific differences regarding the 7-DH-ion pathway of CA.

\section{Materials and Methods}

\section{Bacterial strains and growth conditions}

The strains used were Clostridium scindens ATCC 35704, Clostridium scindens VPI 12708 and Extibacter muris DSM 28561 (SJ24). Bacteria were grown in Brain ㅂeart Infusion Supplement - Salts (BHI-S) medium, consisting of $37 \mathrm{~g} \mathrm{BHI}, 1 \mathrm{~g} \mathrm{~L}$-cysteine, $5 \mathrm{~g}$ yeast extract, $2.5 \mathrm{~g}$ fructose, $50 \mathrm{~mL}$ salts solution $(0.2 \mathrm{~g}$ $\mathrm{CaCl}_{2}, 0.2 \mathrm{MgSO}_{4}, 1 \mathrm{~g} \mathrm{~K}_{2} \mathrm{HPO}_{4}, 1 \mathrm{~g} \mathrm{KH}_{2} \mathrm{PO}_{4}, 10 \mathrm{~g} \mathrm{NAHCO}$ and $2 \mathrm{~g} \mathrm{NaCl}$ per $\mathrm{L}$ of $\mathrm{ddH}_{2} \mathrm{O}$ ) per $\mathrm{LddH}_{2} \mathrm{O}$. The salts solution and media were sterilised by autoclaving. Static growth was carried out at $37^{\circ} \mathrm{C}$ in an anaerobic chamber (Coy Laboratory Products, $95 \% \mathrm{~N}_{2}, 5 \% \mathrm{H}_{2}$ ). A pre-inoculum was prepared from glycerol stocks (using BHIS-S) before inoculating $25 \mathrm{~mL}$ of BHIS-S in Falcon tubes at a starting $\mathrm{OD}_{600}$ of 0.05. Results of the growth curves for the experiments can be found in the Supplementary Information.

In vitro 7-dehydroxylation assays

Bacteria were grown in presence of BAs: CA $(100 \mu \mathrm{M}), \mathrm{CDCA}(200 \mu \mathrm{M}), \alpha \mathrm{MCA}(100 \mu \mathrm{M}), \beta \mathrm{MCA}(100$ $\mu \mathrm{M})$ and UDCA $(100 \mu \mathrm{M})$ or the same volume of ethanol (solvent control). A sterile control (media with ethanol solvent) was also included. Co-induction was performed by adding an additional $100 \mu \mathrm{M}$ 
experiments were conducted at the same time while all the co-induced ones were run simultaneously at a different time. Both experiments included a condition consisting of the amendment of only 100 $\mu \mathrm{M} \mathrm{CA}$, which allows for comparison of the results from co-induced and uninduced experiments. Growth was monitored by periodically measuring the $\mathrm{OD}_{600}$. During the main time points $(0,4,8,12$, 24, 32 and 48 hours), $1 \mathrm{~mL}$ samples were collected for BA extraction in a $2 \mathrm{~mL}$ bead-beating resistant tube. All conditions were performed in triplicates.

\section{Bile acid extraction}

Samples were vacuum dried overnight (ON). Approximately $450 \mathrm{mg}$ of $0.5 \mathrm{~mm}$ zirconium beads were added to the dried samples as well as $500 \mu \mathrm{L}$ ice-cold alkaline acetonitrile (acetonitrile $-25 \%$ ammonia 4:1 $\mathrm{v} / \mathrm{v}$ ) and $100 \mu \mathrm{L}$ of ISTD solution (CA- $\mathrm{d}_{4}$, CDCA- $\mathrm{d}_{4}$, TCA- $\mathrm{d}_{4}$, TUDCA- $_{4}$, DCA- $\mathrm{d}_{4}$ and LCA- $\mathrm{d}_{4}$, each at $100 \mu \mathrm{M}$ in methanol). Samples were homogenised in a Precellys 24 Tissue Homogenizer (Bertin Instruments, Montigny-le-Bretonneux, France) at 6500 rpm 3x 30" beat $30^{\prime \prime}$ rest. Samples were vortexed for 1 hour and centrifugated for 15 minutes at $16000 \mathrm{rcf}$ at room temperature. Approximately $500 \mu \mathrm{L}$ of suspension was carefully collected over the beads level and transferred into a new $1.5 \mathrm{~mL}$ epi tube which was then vacuum dried overnight. Finally, the samples were reconstituted in $1 \mathrm{~mL}$ of ammonium acetate [5mM] - methanol (50:50 v/v) and a 1:20 dilution with the same solvent was prepared in LC-MS glass vials, ready for injection.

\section{RNA extraction and reverse transcription}

$1 \mathrm{~mL}$ of sample was collected in a $15 \mathrm{~mL}$ falcon tube during the mid-log to late-log phase for the RNA extraction. The sample was stored with RNAprotect following the manufacturer protocol (Protocol 5 from RNAprotect Bacteria Reagent Handbook 01/2020, Qiagen) at $-80^{\circ} \mathrm{C}$ until processed. All conditions were performed in triplicates. Lysis and RNA purifications were done using the RNeasy Mini Kit (Qiagen, Hilden, Germany). Bacterial lysis was performed following Protocol 5: Enzymatic Lysis, Proteinase K Digestion and Mechanical Disruption of Bacteria (RNAprotect Bacteria Reagent Handbook 01/2020, Qiagen), with $20 \mu \mathrm{L}$ of proteinase $\mathrm{K}$ for each sample and the required volumes for a number of bacteria $<7.5 \times 10^{8}$. The cell lysis was performed using a Precellys 24 Tissue Homogenizer (Bertin Instruments, Montigny-le-Bretonneux, France) at $6500 \mathrm{rpm} \mathrm{3x} 10$ seconds beat 10 seconds rest. RNA purification was performed following Protocol 7: Purification of Total RNA from Bacterial Lysate using the RNeasy Mini Kit. Centrifugations were carried out at $15000 \mathrm{rcf}$ except for the $2 \mathrm{~min}$ centrifugation which was done at 18000 rcf.

Purified RNA was further subject to a DNase treatment using the RQ DNase I (Promega, Madison, WI, USA) following the manufacturer protocol with small modifications: The final volume was adjusted to a $100 \mu \mathrm{L}$ and incubation was extended to 1 hour at $37^{\circ} \mathrm{C}$. The treated RNA was cleaned-up using the RNeasy Mini Kit (Qiagen, Hilden, Germany) following the RNA Clean-up protocol from the manufacturer (RNeasy Mini Handbook 10/2019) with the 2 min centrifugation done at $18000 \mathrm{rcf}$. Concentration and purity of RNA was measured with a NanoDrop One (Thermo Fisher Scientific, Waltham, MA, USA).

100 ng of RNA was reverse transcribed into CDNA using the GoScript ${ }^{\mathrm{TM}}$ Reverse Transcription Mix, Random Primers (Promega, Madison, WI, USA) following the manufacturer protocol. The process was done in duplicates with one group using water instead of the reaction buffer as a non-reverse transcription control (NRT).

Reverse transcription quantitative $P C R(R T-q P C R)$ 
bioRxiv preprint doi: https://doi.org/10.1101/2022.02.15.480494; this version posted February 17, 2022. The copyright holder for this preprint (which was not certified by peer review) is the author/funder, who has granted bioRxiv a license to display the preprint in perpetuity. It is made available under aCC-BY-NC-ND 4.0 International license.

RT-qPCRs were prepared using the Myra liquid handling system (Bio Molecular Systems, software version 1.6.26) and performed using the Magnetic induction cycler (Mic) platform (Bio Molecular Systems, Upper Coomera, QLD, Australia) with the micPCR software (v2.10.5).

The list of primers used can be found in Supplementary table 2. Samples were prepared with the SensiFAST SYBR No-ROX Kit (Meridian Bioscience, Cincinnati, OH, USA) at a final volume of $10 \mu \mathrm{L}$. All runs were performed with the following program, with small modifications: Initial hold at $95^{\circ} \mathrm{C}$ for 5 minutes with a cycle of $95^{\circ} \mathrm{C}$ for 5 seconds, $54.5^{\circ} \mathrm{C}$ for 20 seconds $\left(54.1^{\circ} \mathrm{C}\right.$ for E. muris SJ24) and $72^{\circ} \mathrm{C}$ for 9 seconds. 40 cycles were done for $C$. scindens ATCC 35704 and 50 for $C$. scindens VPI 12708 and E. muris SJ24. The melting curve, temperature control and acquisition settings were left as default. The quantification was done using three or more reference genes (Supplementary Table 2) based on their expression stability across conditions. NRTs as well as no template controls (NTCs) were included to check for residual DNA or contaminations. Four technical replicates were done for each biological triplicate, totalling at 12 data points per condition. Note that expression data presented in Figure 6 for the CA-only condition (labelled CA) correspond to the pooled expression results (for the condition in which only $100 \mu \mathrm{M}$ Ca was added) from the two sets of experiments presented above (referred to as the co-induced and the uninduced experiments, respectively).

\section{Liquid chromatography - mass spectrometry (LC-MS)}

The quantitative method was performed on an Agilent ultrahigh-performance liquid chromatography 1290 series coupled in tandem to an Agilent 6530 Accurate-Mass Q-TOF mass spectrometer. The separation was done on a Zorbax Eclipse Plus C18 column $(2.1 \times 100 \mathrm{~mm}, 1.8 \mu \mathrm{m})$ and a guard column Zorbax Eclipse Plus C18 $(2.1 \times 5 \mathrm{~mm}, 1.8 \mu \mathrm{m})$ both provided by Agilent technologies (Santa Clara, CA, USA). The column compartment was kept heated at $50^{\circ} \mathrm{C}$. Two different solutions were used as eluents: ammonium acetate $[5 \mathrm{mM}]$ in water as mobile phase $A$ and pure acetonitrile as mobile phase B. A constant flow of $0.4 \mathrm{~mL} / \mathrm{min}$ was maintained over 26 minutes of run time with the following gradient (expressed in eluent B percentage): 0-5.5 min, constant 21.5\% B; 5.5-6 min, 21.5-24.5\% B; 6$10 \mathrm{~min}, 24.5-25 \% \mathrm{~B} ; 10-10.5 \mathrm{~min}, 25-29 \% \mathrm{~B} ; 10.5-14.5 \mathrm{~min}$, isocratic $29 \% \mathrm{~B} ; 14.5-15 \mathrm{~min}, 29-40 \% \mathrm{~B}$; 15-18 min, 40-45\% B; 18-20.5 min, 45-95\% B; 20.5-23 min, constant 95\% B; 23-23.1 min, 95-21.5\% B; $23.10-26 \mathrm{~min}$, isocratic $21.50 \% \mathrm{~B}$. The system equilibration was implemented at the end of the gradient for 3 minutes in initial conditions. The autosampler temperature was maintained at $10^{\circ} \mathrm{C}$ and the injection volume was $5 \mu \mathrm{L}$. The ionisation mode was operated in negative mode for the detection using the Dual AJS Jet stream ESI Assembly. The QTOF acquisition settings were configured in $4 \mathrm{GHz}$ high-resolution mode (resolution $17000 \mathrm{FWHM}$ at $\mathrm{m} / \mathrm{z} 1000$ ), data storage in profile mode and the high-resolution full MS chromatograms were acquired over the range of $\mathrm{m} / \mathrm{z} 100-1700$ at a rate of 3 spectra/s. The mass spectrometer was calibrated in negative mode using ESI-L solution from Agilent technologies every 6 hours to maintain the best possible mass accuracy. Source parameters were setup as follows: drying gas flow, $8 \mathrm{~L} / \mathrm{min}$; gas temperature, $300^{\circ} \mathrm{C}$; nebulizer pressure, $35 \mathrm{psi}$; capillary voltage, 3500V; nozzle voltage, $1000 \mathrm{~V}$. Data were processed afterwards using the MassHunter Quantitative software and MassHunter Qualitative software to control the mass accuracy for each run. In the quantitative method, 42 bile acids were quantified by calibration curves (Supplementary Table 3). The quantification was corrected by addition of internal standards in all samples and calibration levels. Extracted ion chromatograms were generated using a retention time window of \pm $1.5 \mathrm{~min}$ and a mass extraction window of $\pm 30 \mathrm{ppm}$ around the theoretical mass of the targeted bile acid. Unknown BAs were identified when found within the retention time window of a standard with the same ionised mass. Approximate quantification of these unknown BAs was done by using the nearest standard (by retention time) with the same ionised mass. 
Graphpad Prism 9.2.0 (GraphPad) was used to generate the figures shown in this paper and perform pairwise comparisons, the gene expression data was analysed with a linear model (LM) in R language v4.1.2 $2^{55}$ using RStudio ${ }^{56}$. The statistical significance boundary was stablished at a $p$-value $<0.05$.

\section{Conflicts of interest}

The authors declare no conflicts of interest.

\section{Data Availability Statement}

The data used for this manuscript are publicly available in the following link:

https://doi.org/10.5281/zenodo.6034320

\section{References}

1. Monte MJ, Marin JJG, Antelo A, Vazquez-Tato J. Bile acids: Chemistry, physiology, and pathophysiology. World J Gastroenterol 2009; 15:804-16. doi: 10.3748/wjg.15.804

2. Hanafi NI, Mohamed AS, Kadir SHSA, Othman MHD. Overview of bile acids signaling and perspective on the signal of ursodeoxycholic acid, the most hydrophilic bile acid, in the heart. Biomolecules2018; 8. doi: 10.3390/biom8040159

3. Kuipers F, Bloks VW, Groen AK. Beyond intestinal soap - Bile acids in metabolic control. Nat Rev Endocrinol 2014; 10:488-98. doi: 10.1038/nrendo.2014.60

4. Wang H, Chen J, Hollister K, Sowers LC, Forman BM. Endogenous bile acids are ligands for the nuclear receptor FXR/BAR. Mol Cell 1999; 3:543-53. doi: 10.1016/S1097-2765(00)80348-2

5. Bernstein H, Bernstein C, Payne CM, Dvorakova K, Garewal H. Bile acids as carcinogens in human gastrointestinal cancers. Mutat Res - Rev Mutat Res 2005; 589:47-65. doi: 10.1016/j.mrrev.2004.08.001

6. Joyce SA, Gahan CGM. Bile Acid Modifications at the Microbe-Host Interface: Potential for Nutraceutical and Pharmaceutical Interventions in Host Health. Annu Rev Food Sci Technol 2016; 7:313-33. doi: 10.1146/annurev-food-041715-033159

7. Ridlon JM, Kang D-J, Hylemon PB, Ridlon JM, Kang D-J, Hylemon PB. Bile salt biotransformations by human intestinal bacteria. J Lipid Res 2006; 47:241-59. doi: 10.1194/jIr.R500013-JLR200

8. Claudel T, Staels B, Kuipers F. The Farnesoid X receptor: A molecular link between bile acid and lipid and glucose metabolism. Arterioscler. Thromb. Vasc. Biol.2005; 25:2020-31. doi: 10.1161/01.ATV.0000178994.21828.a7

9. Guo C, Chen WD, Wang YD. TGR5, not only a metabolic regulator. Front. Physiol.2016; 7. doi: 10.3389/fphys.2016.00646

10. van Nierop FS, Scheltema MJ, Eggink HM, Pols TW, Sonne DP, Knop FK, Soeters MR. Clinical relevance of the bile acid receptor TGR5 in metabolism. Lancet Diabetes Endocrinol 2016; 5:224-33. doi: 10.1016/S2213-8587(16)30155-3

11. Kawamata Y, Fujii R, Hosoya M, Harada M, Yoshida H, Miwa M, Fukusumi S, Habata Y, Itoh T, Shintani $Y$, et al. A G protein-coupled receptor responsive to bile acids. J Biol Chem 2003; 278:9435-40. doi: 10.1074/jbc.M209706200

12. Brighton CA, Rievaj J, Kuhre RE, Glass LL, Schoonjans K, Holst JJ, Gribble FM, Reimann F. Bile acids trigger GLP-1 release predominantly by accessing basolaterally located $\mathrm{G}$ protein-coupled bile acid receptors. Endocrinology 2015; 156:3961-70. doi: 10.1210/en.2015-1321 
13. Buffie CG, Bucci V, Stein RR, McKenney PT, Ling L, Gobourne A, No D, Liu H, Kinnebrew M, Viale $A$, et al. Precision microbiome reconstitution restores bile acid mediated resistance to Clostridium difficile. Nature 2015; 517:205-8. doi: 10.1038/nature13828

14. Studer N, Desharnais L, Beutler M, Brugiroux S, Terrazos MA, Menin L, Schürch CM, McCoy KD, Kuehne SA, Minton NP, et al. Functional intestinal bile acid $7 \alpha$-dehydroxylation by Clostridium scindens associated with protection from Clostridium difficile infection in a gnotobiotic mouse model. Front Cell Infect Microbiol 2016; 6. doi: 10.3389/fcimb.2016.00191

15. Li J, Dawson PA. Animal models to study bile acid metabolism. Biochim. Biophys. Acta - Mol. Basis Dis.2019; 1865:895-911. doi: 10.1016/j.bbadis.2018.05.011

16. Honda A, Miyazaki T, Iwamoto J, Hirayama T, Morishita $\mathrm{Y}$, Monma T, Ueda H, Mizuno S, Sugiyama F, Takahashi S, et al. Regulation of bile acid metabolism in mouse models with hydrophobic bile acid composition. J Lipid Res 2020; 61:54-69. doi: 10.1194/jlr.RA119000395

17. Morris GN, Winter J, Cato EP, Ritchie AE, Bokkenheuser VD. Clostridium scindens sp. nov., a Human Intestinal Bacterium with Desmolytic Activity on Corticoids. Int J Syst Bacteriol 1985; 35:478-81. doi: 10.1099/00207713-35-4-478

18. Lagkouvardos I, Pukall R, Abt B, Foesel BU, Meier-Kolthoff JP, Kumar N, Bresciani A, Martínez I, Just $\mathrm{S}$, Ziegler $\mathrm{C}$, et al. The Mouse Intestinal Bacterial Collection (miBC) provides host-specific insight into cultured diversity and functional potential of the gut microbiota. Nat Microbiol 2016; 1:1-15. doi: 10.1038/nmicrobiol.2016.131

19. Streidl T, Kumar N, Suarez LN, Rohn S, Clavel T. Extibacter. In: Bergey's Manual of Systematics of Archaea and Bacteria. American Cancer Society; 2019. page 1-7.doi: 10.1002/9781118960608.gbm01749

20. Marion S, Studer N, Desharnais L, Menin L, Escrig S, Meibom A, Hapfelmeier S, Bernier-Latmani R. In vitro and in vivo characterization of Clostridium scindens bile acid transformations. Gut Microbes 2019; 10:481-503. doi: 10.1080/19490976.2018.1549420

21. Mallonee DH, White WB, Hylemon PB. Cloning and sequencing of a bile acid-inducible operon from Eubacterium sp. strain VPI 12708. J Bacteriol 1990; 172:7011-9.

22. Ridlon JM, Hylemon PB. Identification and characterization of two bile acid coenzyme A transferases from Clostridium scindens, a bile acid $7 \alpha$-dehydroxylating intestinal bacterium . J Lipid Res 2012; 53:66-76. doi: 10.1194/jIr.m020313

23. Streidl T, Karkossa I, Segura Muñoz RR, Eberl C, Zaufel A, Plagge J, Schmaltz R, Schubert K, Basic $M$, Schneider KM, et al. The gut bacterium Extibacter muris produces secondary bile acids and influences liver physiology in gnotobiotic mice. Gut Microbes 2021; 13:1-21. doi: 10.1080/19490976.2020.1854008

24. Wells JE, Hylemon PB. Identification and Characterization of a Bile Acid $7 \alpha$-Dehydroxylation Operon in Clostridium sp. Strain TO-931, a Highly Active 7 $\alpha$-Dehydroxylating Strain Isolated from Human Feces. Appl Environ Microbiol 2000; 66:1107-13. doi: 10.1128/AEM.66.3.11071113.2000

25. Funabashi M, Grove TL, Wang M, Varma Y, McFadden ME, Brown LC, Guo C, Higginbottom S, Almo SC, Fischbach MA. A metabolic pathway for bile acid dehydroxylation by the gut microbiome. Nature 2020; :1-5. doi: 10.1038/s41586-020-2396-4

26. Macdonald IA, Hutchison DM. Epimerization versus dehydroxylation of the $7 \alpha$-hydroxyl-group of primary bile acids: Competitive studies with Clostridium absonum and $7 \alpha$-dehydroxylating bacteria (Eubacterium SP.). J Steroid Biochem 1982; 17:295-303. doi: 10.1016/0022- 


\section{1(82)90203-5}

27. Doerner KC, Takamine F, LaVoie CP, Mallonee DH, Hylemon PB. Assessment of fecal bacteria with bile acid $7 \alpha$-dehydroxylating activity for the presence of bai-like genes. Appl Environ Microbiol 1997; 63:1185-8.

28. Vinithakumari AA, Hernandez BG, Ghimire S, Adams S, Stokes C, Jepsen I, Brezina C, Sahin O, Li $G$, Tangudu $C$, et al. A model screening pipeline for bile acid converting anti-Clostridioides difficile bacteria reveals unique biotherapeutic potential of Peptacetobacter hiranonis. bioRxiv 2021; :2021.09.29.462466. doi: 10.1101/2021.09.29.462466

29. Ridlon JM. Enzymology and molecular biology of bile acid 7alpha- and 7beta- dehydroxylation by the intestinal bacteria Clostridium scindens and Clostridium hylemonae. PhD Thesis Virginia Commonw Univ 2008; :1-333. doi: https://doi.org/10.25772/BN62-H676

30. Makino I, Nakagawa S. Changes in biliary lipid and biliary bile acid composition in patients after administration of ursodeoxycholic acid. J Lipid Res 1978; 19:723-8. doi: 10.1016/s00222275(20)41272-6

31. Meir K, Kitsberg D, Alkalay I, Szafer F, Rosen H, Shpitzen S, Avi L Ben, Staels B, Fievet C, Meiner $V$, et al. Human sterol 27-hydroxylase (CYP27) overexpressor transgenic mouse model. Evidence against 27-hydroxycholesterol as a critical regulator of cholesterol homeostasis. J Biol Chem 2002; 277:34036-41. doi: 10.1074/jbc.M201122200

32. Devendran S, Shrestha R, Alves JMP, Wolf PG, Ly L, Hernandez AG, Méndez-García C, Inboden A, Wiley J, Paul O, et al. Clostridium scindens ATCC 35704: Integration of nutritional requirements, the complete genome sequence, and global transcriptional responses to bile acids. Appl Environ Microbiol 2019; 85. doi: 10.1128/AEM.00052-19

33. Ridlon JM, Devendran S, Alves JM, Doden H, Wolf PG, Pereira G V., Ly L, Volland A, Takei H, Nittono $\mathrm{H}$, et al. The 'in vivo lifestyle' of bile acid $7 \alpha$-dehydroxylating bacteria: comparative genomics, metatranscriptomic, and bile acid metabolomics analysis of a defined microbial community in gnotobiotic mice. Gut Microbes 2019; :1-24. doi: 10.1080/19490976.2019.1618173

34. Bhowmik S, Jones DH, Chiu HP, Park IH, Chiu HJ, Axelrod HL, Farr CL, Tien HJ, Agarwalla S, Lesley SA. Structural and functional characterization of BaiA, an enzyme involved in secondary bile acid synthesis in human gut microbe. Proteins Struct Funct Bioinforma 2014; 82:216-29. doi: 10.1002/prot.24353

35. Ridlon JM, Harris SC, Bhowmik S, Kang D-J, Hylemon PB. Consequences of bile salt biotransformations by intestinal bacteria. Gut Microbes 2016; 7:22-39. doi: 10.1080/19490976.2015.1127483

36. Heinken A, Ravcheev DA, Baldini F, Heirendt L, Fleming RMTT, Thiele I. Systematic assessment of secondary bile acid metabolism in gut microbes reveals distinct metabolic capabilities in inflammatory bowel disease. Microbiome 2019; 7:75. doi: 10.1186/s40168-019-0689-3

37. Hofmann AF, Hagey LR. Key discoveries in bile acid chemistry and biology and their clinical applications: History of the last eight decades. J. Lipid Res.2014; 55:1553-95. doi: 10.1194/jlr.R049437

38. Winston JA, Theriot CM. Diversification of host bile acids by members of the gut microbiota. Gut Microbes2020; 11:158-71. doi: 10.1080/19490976.2019.1674124

39. Ridlon JM, Kang DJ, Hylemon PB. Isolation and characterization of a bile acid inducible $7 \alpha-$ dehydroxylating operon in Clostridium hylemonae TN271. Anaerobe 2010; 16:137-46. doi: 
40. Song I, Gotoh Y, Ogura Y, Hayashi T, Fukiya S, Yokota A. Comparative genomic and physiological analysis against Clostridium scindens reveals Eubacterium sp. C-25 as an atypical deoxycholic acid producer of the human gut microbiota. Microorganisms 2021; 9:2254. doi: 10.3390/microorganisms9112254

41. Hylemon PB, Melone PD, Franklund C V., Lund E, Bjorkhem I. Mechanism of intestinal 7 $\alpha-$ dehydroxylation of cholic acid: Evidence that allo-deoxycholic acid is an inducible side-product. J Lipid Res 1991; 32:89-96.

42. Masuda N, Oda H, Hirano S, Tanaka H. Enhancement of the $7 \alpha$-dehydroxylase activity of a gram-positive intestinal anaerobe by flavins. Appl Environ Microbiol 1983; 45:308-9.

43. Kitahara M, Takamine F, Imamura T, Benno Y. Assignment of Eubacterium sp. VPI 12708 and related strains with high bile acid $7 \alpha$-dehydroxylating activity to Clostridium scindens and proposal of Clostridium hylemonae sp. nov., isolated from human faeces. Int J Syst Evol Microbiol 2000; 50:971-8. doi: 10.1099/00207713-50-3-971

44. Benno Y, Kitahara M, Takamine F, Imamura T. Clostridium hiranonis sp. nov., a human intestinal bacterium with bile acid 7 $\alpha$-dehydroxylating activity. Int J Syst Evol Microbiol 2001; 51:39-44. doi: 10.1099/00207713-51-1-39

45. Abdallah E, Emile SH, Elfeki H, Fikry M, Abdelshafy M, Elshobaky A, Elgendy H, Thabet W, Youssef $\mathrm{M}$, Elghadban $\mathrm{H}$, et al. Role of ursodeoxycholic acid in the prevention of gallstone formation after laparoscopic sleeve gastrectomy. Surg Today 2017; 47:844-50. doi: 10.1007/s00595-016-1446-x

46. Coleman JP, Hudson LL, Adams MJ. Characterization and regulation of the NADP-linked 7 $\alpha-$ hydroxysteroid dehydrogenase gene from Clostridium sordellii. J Bacteriol 1994; 176:4865-74.

47. Ferrandi EE, Bertolesi GM, Polentini F, Negri A, Riva S, Monti D. In search of sustainable chemical processes: Cloning, recombinant expression, and functional characterization of the $7 \alpha-$ and $7 \beta$-hydroxysteroid dehydrogenases from Clostridium absonum. Appl Microbiol Biotechnol 2012; 95:1221-33. doi: 10.1007/s00253-011-3798-x

48. Devlin AS, Fischbach MA. A biosynthetic pathway for a prominent class of microbiota-derived bile acids. Nat Chem Biol 2015; 11:685-90. doi: 10.1038/nchembio.1864

49. Sayin SI, Wahlström A, Felin J, Jäntti S, Marschall HU, Bamberg K, Angelin B, Hyötyläinen T, Orešič $M, B a ̈ c k h e d ~ F$. Gut microbiota regulates bile acid metabolism by reducing the levels of tauro-beta-muricholic acid, a naturally occurring FXR antagonist. Cell Metab 2013; 17:225-35. doi: 10.1016/j.cmet.2013.01.003

50. Stellwag EJ, Hylemon PB. $7 \alpha$-Dehydroxylation of cholic acid and chenodeoxycholic acid by Clostridium leptum. J Lipid Res 1979; 20:325-33.

51. Narushima S, Itoh K, Miyamoto Y, Park SH, Nagata K, Kuruma K, Uchida K. Deoxycholic acid formation in gnotobiotic mice associated with human intestinal bacteria. Lipids 2006; 41:83543. doi: 10.1007/s11745-006-5038-1

52. Eyssen HJ, Parmentier GG, Mertens JA. Sulfated Bile Acids in Germ-Free and Conventional Mice. Eur J Biochem 1976; 66:507-14. doi: 10.1111/j.1432-1033.1976.tb10576.x

53. Han J, Liu Y, Wang R, Yang J, Ling V, Borchers $\mathrm{CH}$. Metabolic profiling of bile acids in human and mouse blood by LC-MS/MS in combination with phospholipid-depletion solid-phase extraction. Anal Chem 2015; 87:1127-36. doi: 10.1021/ac503816u 
bioRxiv preprint doi: https://doi.org/10.1101/2022.02.15.480494; this version posted February 17, 2022. The copyright holder for this preprint (which was not certified by peer review) is the author/funder, who has granted bioRxiv a license to display the preprint in perpetuity. It is made available under aCC-BY-NC-ND 4.0 International license.

54. Straniero S, Laskar A, Savva C, Härdfeldt J, Angelin B, Rudling M. Of mice and men: Murine bile acids explain species differences in the regulation of bile acid and cholesterol metabolism. J Lipid Res 2020; 61:480-91. doi: 10.1194/jlr.RA119000307

55. Team RC. R: A language and environment for statistical computing. 2021;

56. RStudio Team (2020). RStudio: Integrated Development for R. 2020;

\section{Figure Legends}

Figure 1. List of deconjugated human and rodent bile acids (BAs) discussed here. The characteristic that distinguishes BAs is the presence of a hydroxyl group at the C-3, C-6, C-7 and/or C-12 position. The hydroxyl groups can be in $\alpha-$ or $\beta$ conformation, oxidised into a ketone group, or fully removed (dehydroxylated BA). CA and CDCA are primary BAs of both humans and rodents whereas MCAs are exclusively produced by rodents. $\left({ }^{*}\right)$ UDCA is a primary BA in rodents while it is a secondary BA in humans where the gut microbes epimerise it from CDCA. ( + ) Bile acids that might have been detected in this study but for which no standards were available. (¥) Also known as 6-oxolithocholic acid (6-oxoLCA). See Supplementary Table 3 for full chemical names of the BAs.

Figure 2. In vitro 7-dehydroxylation of cholic acid. The 7-dehydroxylation of CA was tested in (A) Clostridium scindens ATCC 35704, (B) C. scindens VPI 12708 and (C) Extibacter muris DSM 28561 (SJ24) over time. All strains were grown anaerobically in BHIS-S containing $100 \mu \mathrm{M}$ CA. Bile acids were extracted from the suspended biomass. Error bars represent the standard deviation of the mean of biological triplicates.

Figure 3. In vitro $7 \alpha$-dehydroxylation of CDCA. The transformation of CDCA into secondary bile acids was tested with and without co-induction with $100 \mu \mathrm{M}$ of ${ }^{13} \mathrm{C}$-CA. $200 \mu \mathrm{M}$ of CDCA were used based on previous experiments by Marion et al. (A) Clostridium scindens ATCC 35704, (B) C. scindens VPI 12708 and (C) E. muris DSM 28561 (SJ24). Bile acids were extracted from the suspended biomass. Error bars represent the standard deviation of the mean of biological triplicates.

Figure 4. In vitro 7-dehydroxylation of UDCA. The transformation of $100 \mu \mathrm{M}$ of UDCA into secondary bile acids was tested with and without co-induction with $100 \mu \mathrm{M}$ of ${ }^{13} \mathrm{C}$-CA. (A) Clostridium scindens ATCC 35704, (B) C. scindens VPI 12708 and (C) Extibacter muris DSM 28561 (SJ24) were grown anaerobically in BHIS-S. Bile acids were extracted from suspended biomass. Two compounds were detected that could not be identified due to missing standards but their oxidative state can be estimated based on their ionised mass. X-oxoUDCA had the same mass as other bile acids with one ketone group and one hydroxyl group. The other unidentified compound had the same mass as UDCA and therefore it is likely to be an isoform with a $3 \beta$ conformation. The retention times for these compounds was unique and therefore could not be identified further. Concentration values of the unknown BAs could only be estimated for the same reason. Error bars represent the standard deviation of the mean of biological triplicates.

Figure 5. In vitro 7-dehydroxylation of $\alpha \mathrm{MCA}$. The transformation of $100 \mu \mathrm{M}$ of $\alpha \mathrm{MCA}$ into secondary bile acids was tested with and without co-induction with $100 \mu \mathrm{M}$ of ${ }^{13} \mathrm{C}$-CA. (A) Clostridium scindens ATCC 35704, (B) C. scindens VPI 12708 and (C) Extibacter muris DSM 28561 (SJ24) were grown anaerobically in BHIS-S. Bile acids were extracted from suspended biomass. Several compounds were detected that could not be identified due to missing standards but their oxidative state can be estimated based on their ionised mass. X- or Y- oxoaMCA had the same mass as other bile acids with one ketone group and two hydroxyl groups. The other unidentified compound had the same mass as secondary bile acids that have been dehydroxylated $(-1-\mathrm{OH})$, have one ketone group and one hydroxyl group. The retention times for these compounds did not correspond to that of any known standard. Concentration values of the unknown BAs could only be semiquantitative for the same reason. Error bars represent the standard deviation of the mean of biological triplicates.

Figure 6. bai gene expression in the presence of CA (first panel) or in that of various primary BAs either uninduced or and co-induced with ${ }^{13} \mathrm{C}$-CA. Data for the CA-only condition correspond to the pooled expression results from the two sets of experiments (referred to as uninduced and co-induced) that both include this condition, as described in the text. The expression (normalised to at least three reference genes) is relative to the no BA condition including an equivalent volume of solvent (ethanol). (A) Clostridium scindens ATCC 35704, (B) C. scindens VPI 12708 and (C) Extibacter muris DSM 28561 (SJ24) gene expression of baiCD, baiE (part of the bai operon), and accessory genes baiJ and baiO (only in E. muris DSM SJ24) was measured. CA, UDCA, $\alpha M C A, \beta M C A$ and the co-inducing ${ }^{13} C$-CA were used at $100 \mu \mathrm{M}$, CDCA was used at 200 $\mu \mathrm{M}$. A detailed view of the $C$. scindens VPI 12708 uninduced vs 13C-CA co-induced in the CDCA group is found in 
bioRxiv preprint doi: https://doi.org/10.1101/2022.02.15.480494; this version posted February 17, 2022. The copyright holder for this preprint (which was not certified by peer review) is the author/funder, who has granted bioRxiv a license to display the preprint in perpetuity. It is made available under aCC-BY-NC-ND 4.0 International license. replicates. Some error bars may look elongated due to the logarithmic scale of the $Y$ axis. $\left({ }^{* * *}\right)$ indicates a $p$-value $<0.001$ in a linear model analysis comparing the uninduced vs ${ }^{13} \mathrm{C}$-CA co-induced factor for each bai gene. 


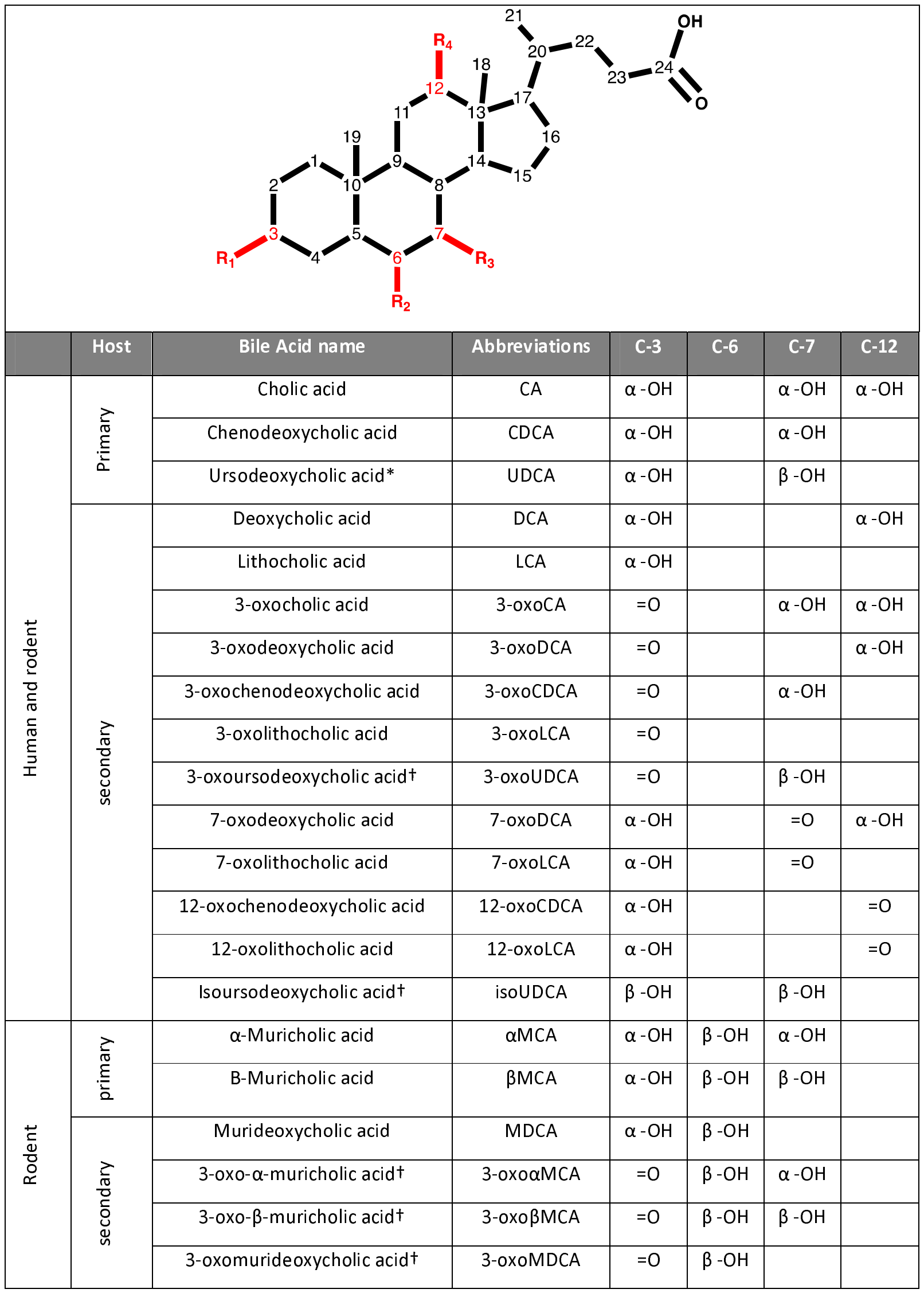




\begin{tabular}{|c|c|c|c|c|c|c|}
\hline & & 6-oxo- $\alpha$-muricholic acid $\dagger$ & 6-oxoaMCA & $\alpha-\mathrm{OH}$ & $=0$ & $\alpha-\mathrm{OH}$ \\
\hline & & 6-oxo- $\beta$-muricholic acid $\dagger$ & 6-oxoßMCA & $\alpha-\mathrm{OH}$ & $=0$ & $\beta-\mathrm{OH}$ \\
\hline & & 6-oxomurideoxycholic acid $\neq$ & 6-oxoMDCA & $\alpha-\mathrm{OH}$ & $=0$ & \\
\hline & & 7-oxomurideoxycholic acid + & 7-oxoMDCA & $\alpha-\mathrm{OH}$ & $\beta-\mathrm{OH}$ & $=0$ \\
\hline & & $\omega$-Muricholic acidt & $\omega M C A$ & $\alpha-\mathrm{OH}$ & $\alpha-\mathrm{OH}$ & $\beta-\mathrm{OH}$ \\
\hline
\end{tabular}


A)

C. scindens ATCC 35704

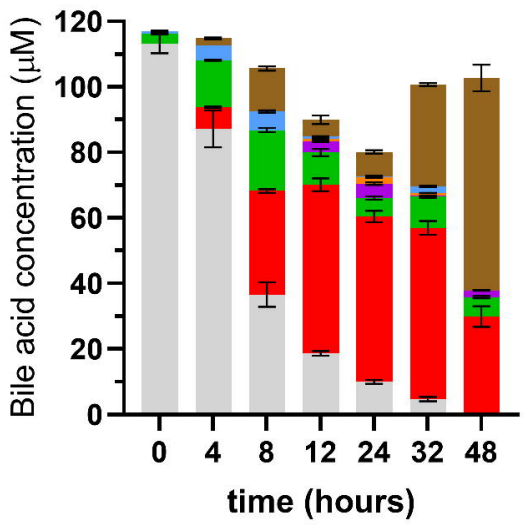

CA

B)

C. scindens VPI 12708

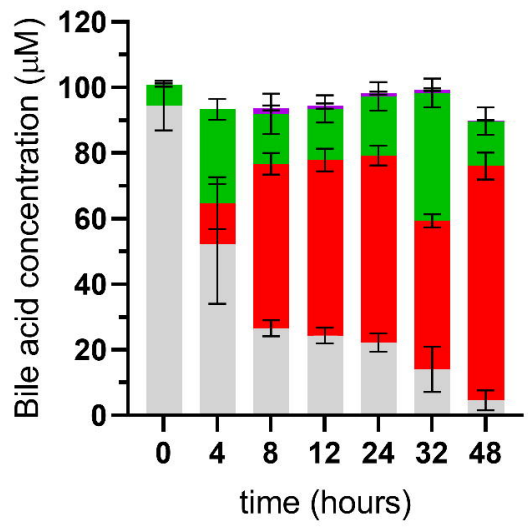

CA

DCA

7-oxoDCA

3-oxoDCA

c)

E. muris SJ24

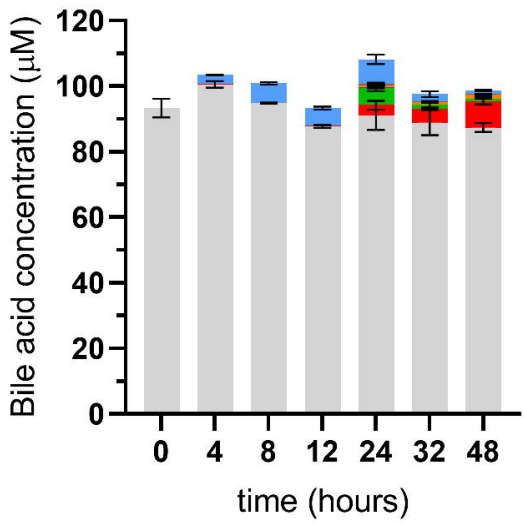

CA

DCA

7-oxoDCA

- 3-oxoDCA

3-oxoCA

12-oxoCDCA

12-oxoLCA 
C. scindens ATCC 35704 uninduced

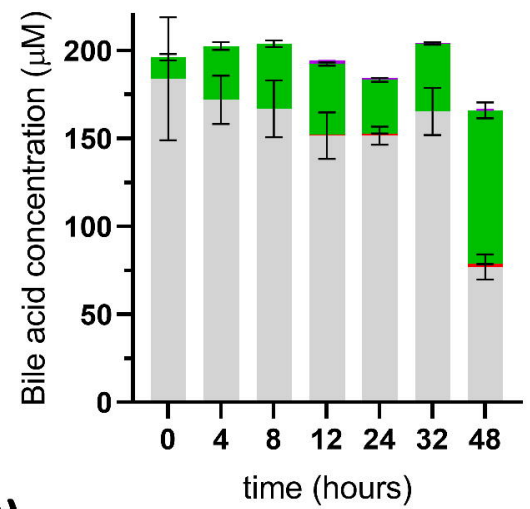

B)

C. scindens VPI 12708 uninduced

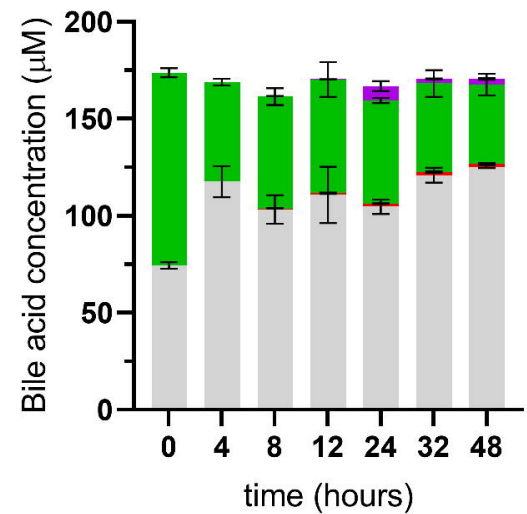

C)

E. muris SJ24 uninduced

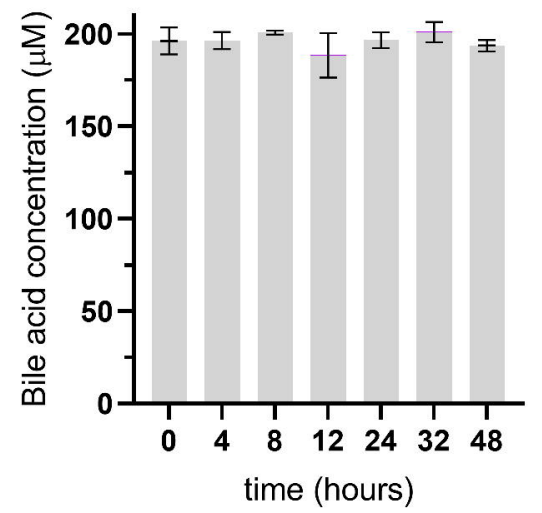

C. scindens ATCC $35704{ }^{13} \mathrm{C}$-CA co-induced

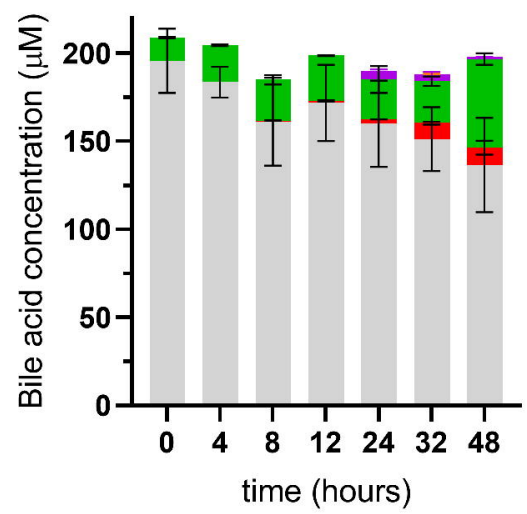

C. scindens VPI $12708{ }^{13} \mathrm{C}$-CA co-induced

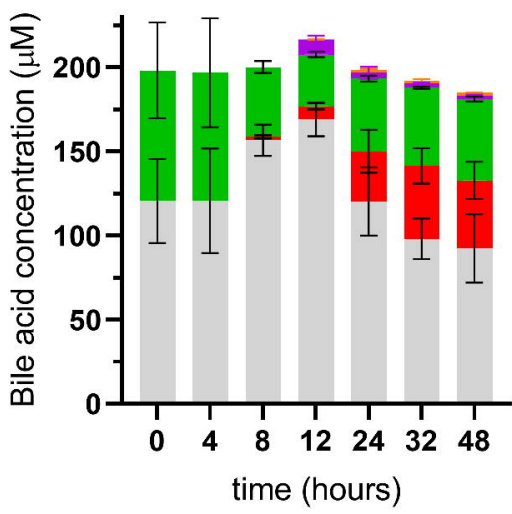

- CDCA

- LCA

7-oxoLCA

3-oxoCDCA

3-oxoLCA
E. muris SJ24 ${ }^{13} \mathrm{C}$-CA co-induced

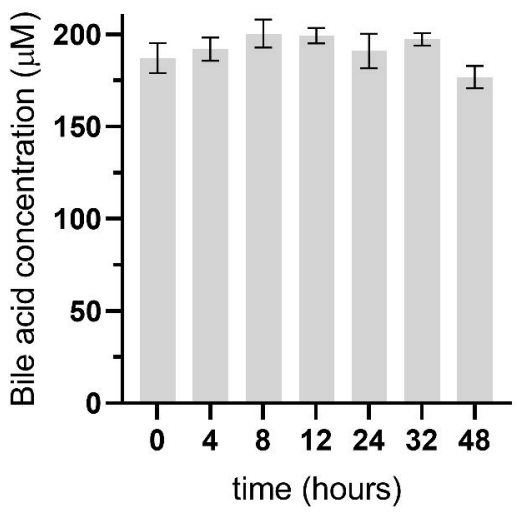

CDCA 
A)

C. scindens ATCC 35704 uninduced

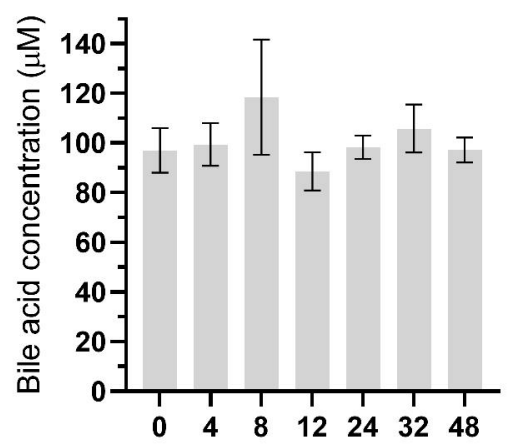

B)
C. scindens VPI 12708 uninduced

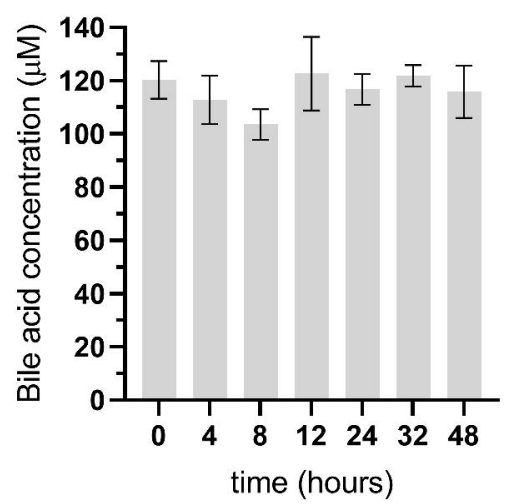

C)

E. muris SJ24 uninduced

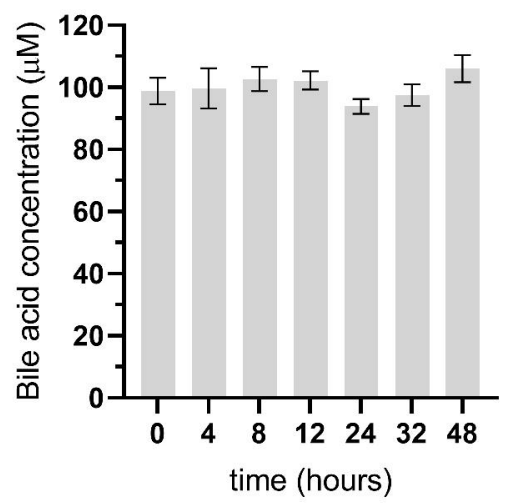

C. scindens ATCC $35704{ }^{13} \mathrm{C}$-CA co-induced

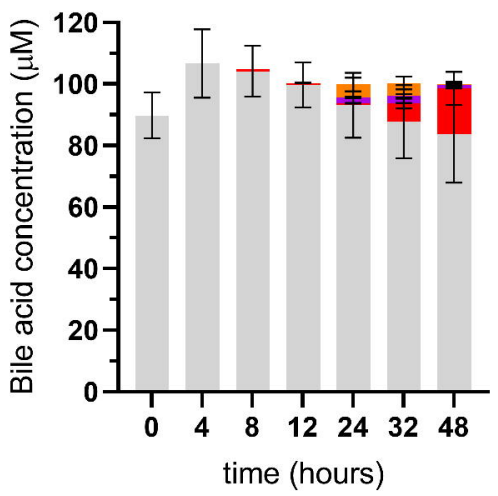

UDCA

- LCA

3-oxoLCA

- X-oxoUDCA

Potential isoform of UDCA
C. scindens VPI $12708{ }^{13} \mathrm{C}$-CA co-induced

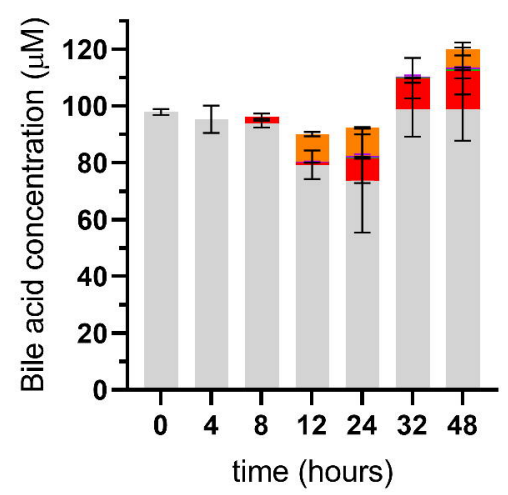

E. muris $\mathrm{SJ} 24{ }^{13} \mathrm{C}$-CA co-induced

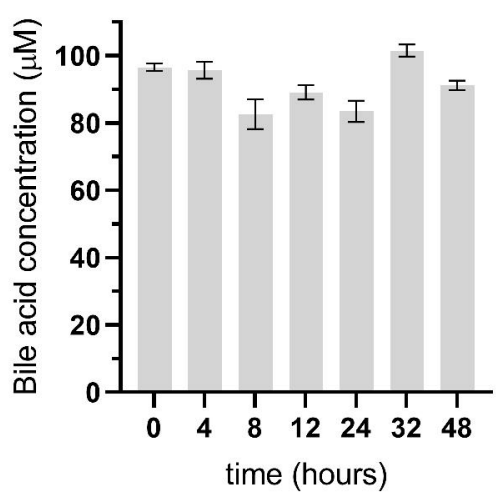


C. scindens ATCC 35704 uninduced

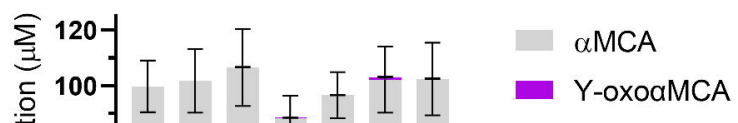

B)

\section{C. scindens VPI 12708 uninduced}

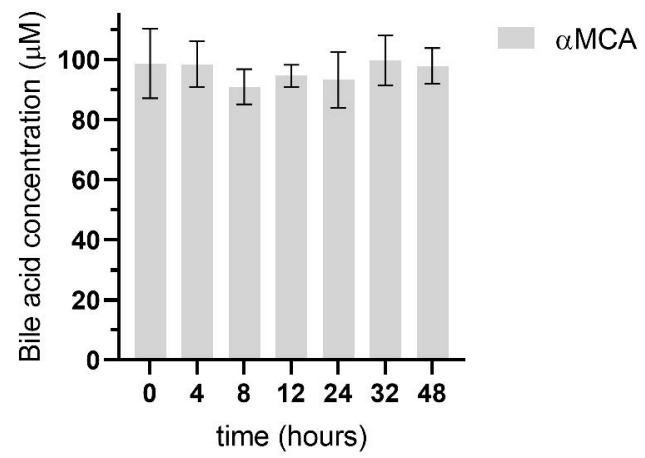

C)

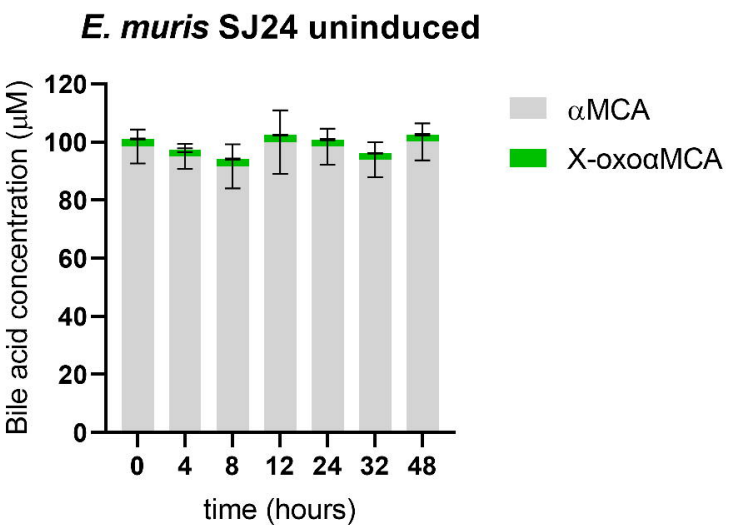

C. scindens ATCC $35704{ }^{13} \mathrm{C}$-CA co-induced

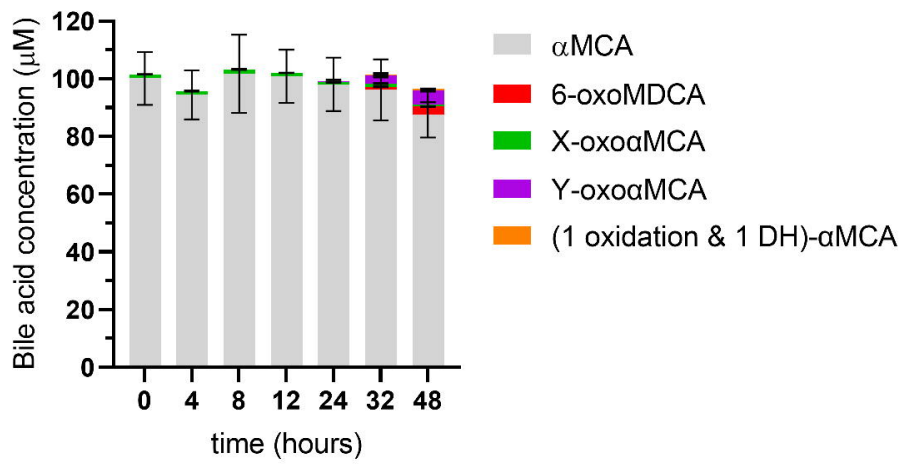

C. scindens VPI $12708{ }^{13} \mathrm{C}$-CA co-induced

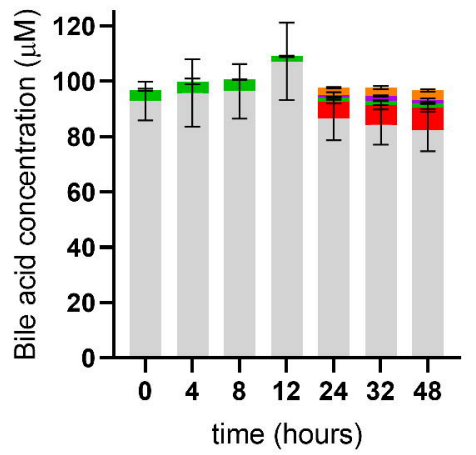

$\alpha \mathrm{MCA}$

6-oxoMDCA

X-oxoaMCA

- Y-oxoaMCA

(1 oxidation \& $1 \mathrm{DH})-\alpha M C A$
E. muris SJ24 ${ }^{13} \mathrm{C}$-CA co-induced

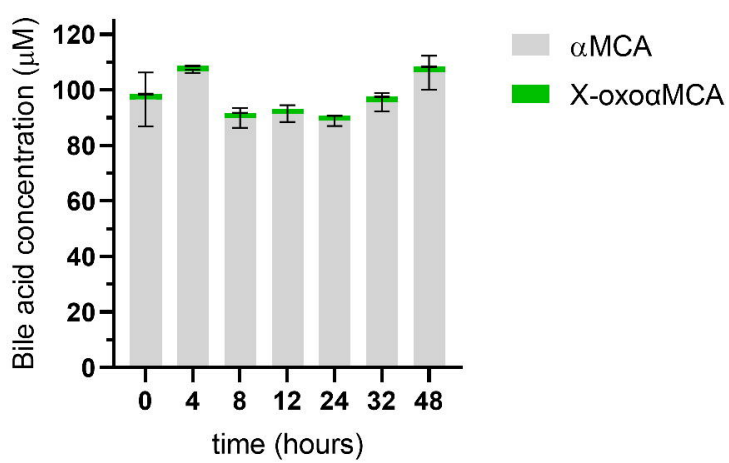


A)

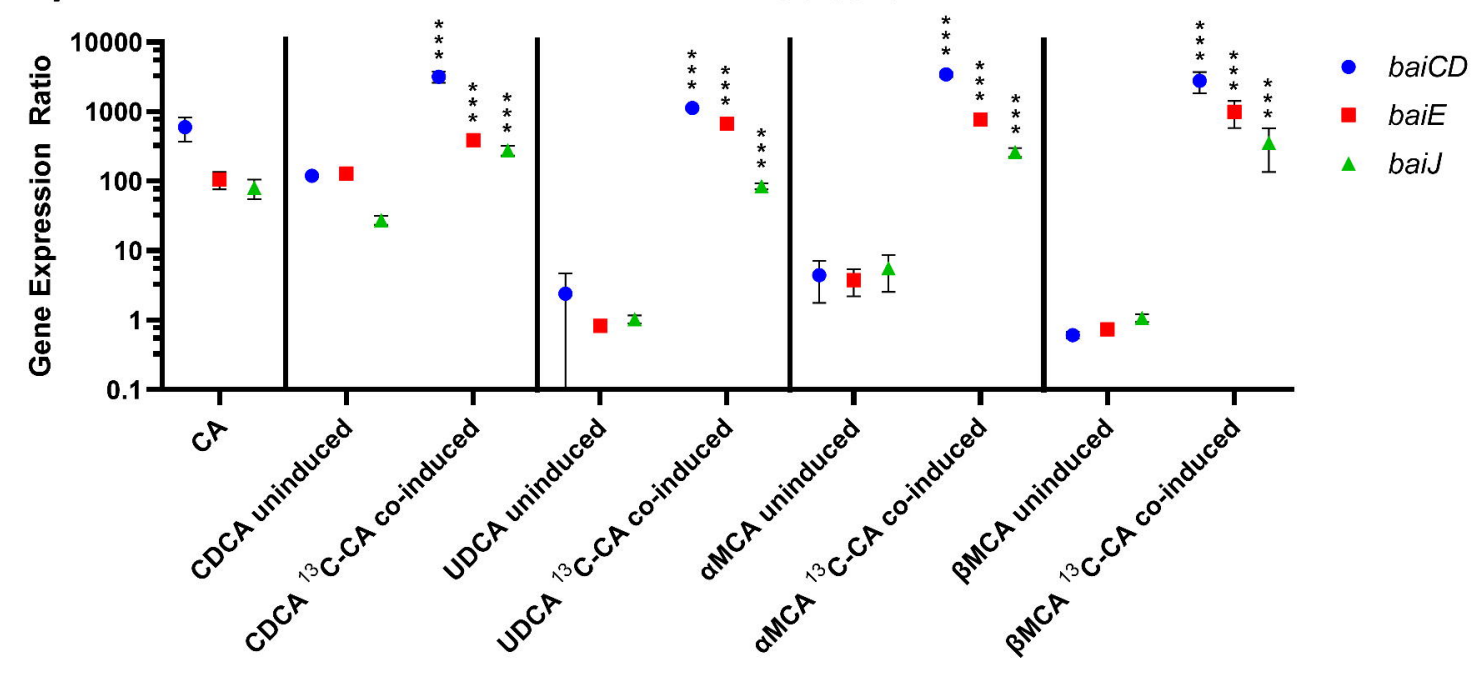

B)

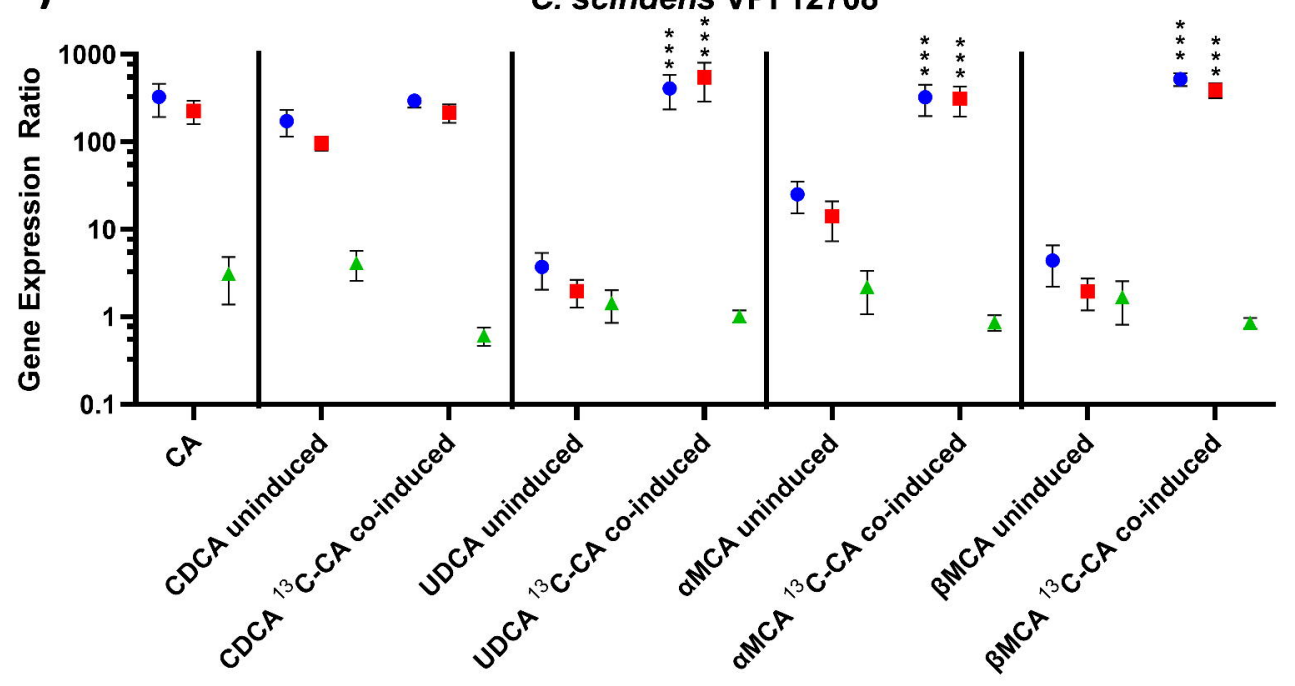

C)

E. muris SJ24

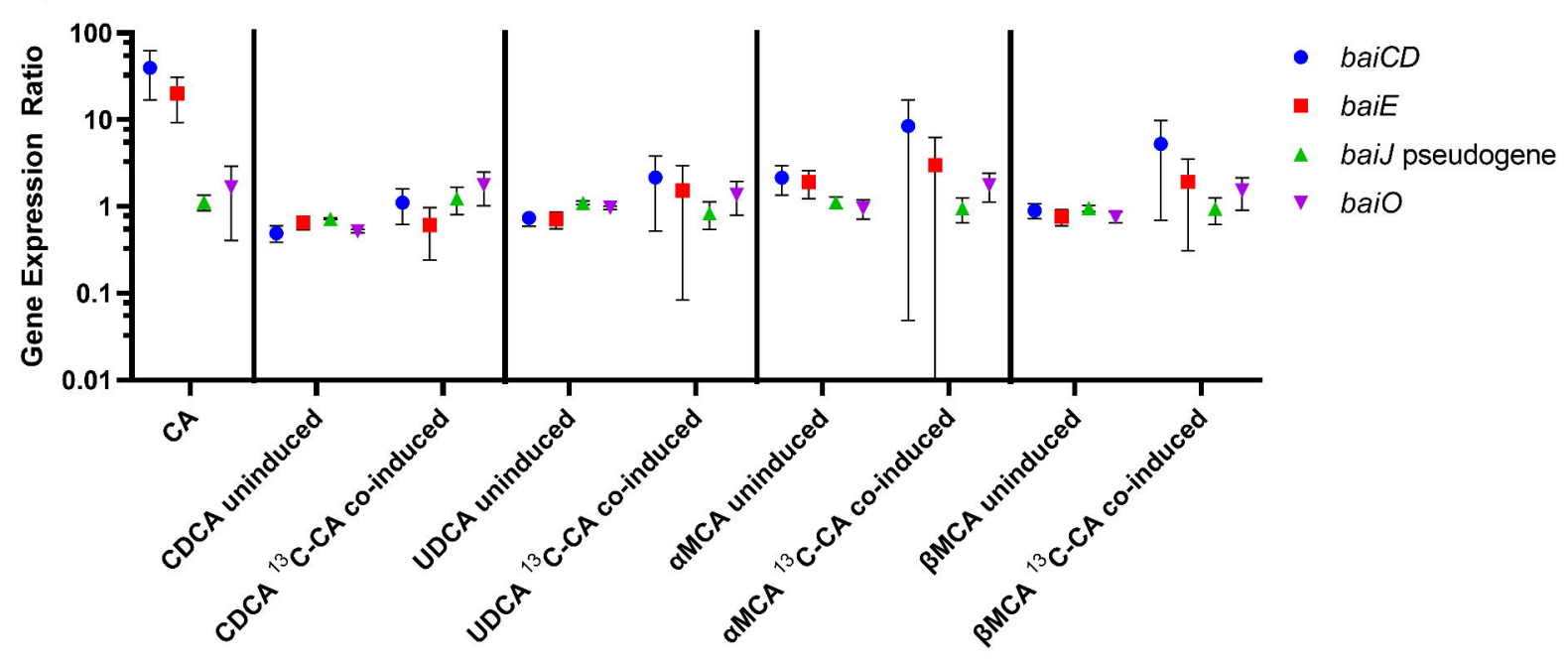

